\title{
Total superior vena cava reconstruction guided by preoperative three-dimensional (3D)-computed tomography bronchography and angiography
}

\author{
Zhenyang Zhang ${ }^{1 \#}$, Jiangbo Lin ${ }^{1}$, Tianci Chai ${ }^{1 \#}$, Mingqiang Kang ${ }^{1}$, Wenhua Chen ${ }^{2}$, Hanfan Qiu ${ }^{3}$, Zhiqiang \\ Zou $^{4}$, Lei Gao ${ }^{1}$, Chuangcai Yang ${ }^{1}$, Jiafu Zhu ${ }^{1}$ \\ ${ }^{1}$ Department of Thoracic Surgery, ${ }^{2}$ Department of Anesthesiology, ${ }^{3}$ Department of Cardiac Surgery, ${ }^{4}$ Department of Intensive Care Unit, Fujian \\ Medical University Union Hospital, Fuzhou, China \\ Contributions: (I) Conception and design: J Lin, M Kang; (II) Administrative support: J Lin; (III) Provision of study materials or patients: Z Zhang, C \\ Yang; (IV) Collection and assembly of data: W Chen, H Qiu, Z Zou; (V) Data analysis and interpretation: J Zhu, L Gao; (VI) Manuscript writing: All \\ authors; (VII) Final approval of manuscript: All authors. \\ \#These authors contributed equally to this work. \\ Correspondence to: Lin Jiangbo. Department of Thoracic Surgery, Fujian Medical University Union Hospital, Fuzhou, China. Email: jiangbolin8009@sina.com.
}

\begin{abstract}
Background: The treatment of superior vena cava syndrome caused by invasive thymoma is challenging. This paper aims to explore the application of preoperative three-dimensional computed tomography bronchography and angiography (3D-CTBA) for total superior vena cava reconstruction.

Methods: Total superior vena cava reconstruction guided by preoperative 3D-CTBA in the treatment of superior vena cava syndrome offers more accurate surgical evaluation and more effective procedure of multidisciplinary team (MDT), assists radical dissection and vascular reconstruction as planed in the way of "Step by Step". It also makes the follow-up procedure more effective.

Results: High-quality thoracic computed tomography (CT) image is essential. A medical team ensures procedural success with 3D-CTBA. Using this approach, five patients have been treated successfully. The average operative length was 324 minutes and the average blood loss was $190 \mathrm{~mL}$. There was no surgical mortality. Five patients are alive.

Conclusions: Total superior vena cava reconstruction guided by preoperative 3D-CTBA is an effective technology for radical resection of mediastinal lesions combined with artificial vascular replacement. Meanwhile, 3D-CTBA improves the efficiency of MDT and surgical planning. It contributes to alleviate symptoms of SVCS and improve the quality of postoperative life.
\end{abstract}

Keywords: Total superior vena cava replacement; 3D-CTBA; superior vena cava syndrome; anterior mediastinal thymic tumor

Submitted Oct 22, 2019. Accepted for publication Mar 03, 2020.

doi: $10.21037 /$ tcr-19-2249

View this article at: http://dx.doi.org/10.21037/tcr-19-2249

\section{Introduction}

The invasion of anterior mediastinal tumor on superior vena cava (SVC) is the main reason of superior vena cava obstruction syndrome. Expanded excision of mediastinal lesions and superior vena cava system, including SVC, left brachiocephalic vein (LBCV) and right brachiocephalic vein (RBCV), combined with artificial vascular reconstruction is an effective way for treating the disease $(1,2)$. With the application of 3D-CTBA reconstruction in many clinical fields, especially its guidance in preoperative planning and intraoperative operation for anatomic pulmonary segmentectomy has been discussed $(3,4)$. However, the application of 3D-CTBA in the process of superior vena cava replacement has hardly been discussed. The process of 
Table 1 General data of patients in this group and 3D reconstruction information

\begin{tabular}{lccccccccc}
\hline Case & Gender & Age & Pathology & LLli & LRli & LSVCi & TMD $(\mathrm{cm})$ & L/R UEVP(cm) & ISIV \\
\hline 1 & M & 60 & B2 thymoma & 4 & 2 & 3 & 6.5 & $28 / 25$ & No \\
2 & M & 62 & AB thymoma & 5 & 2 & 4 & 8.0 & $30 / 32$ & No \\
3 & $\mathrm{~F}$ & 48 & Thymic carcinoma & 3 & 3 & 3 & 6.0 & $26 / 22$ & No \\
4 & $\mathrm{M}$ & 45 & B3 thymoma & 2 & 2 & 3 & 5.0 & $24 / 24$ & No \\
5 & $\mathrm{~F}$ & 64 & B2 thymoma & 2 & 3 & 2 & 5.5 & $25 / 26$ & Yes \\
Mean & & 55.8 & & 3.2 & 2.4 & 3 & 6.2 & $26.6 / 25.8$ & \\
$\mathrm{~s}$ & & & 1.17 & 0.49 & 0.63 & 1.03 & $2.15 / 3.37$ & \\
\hline
\end{tabular}

LII, length of left innominate vein invaded; LSVCI, length of superior vena cava invaded; L/R UEVP(cm), left/right upper extremity venous pressure(cm); ISIV, whether the subclavian vein and the internal jugular vein was invaded; TMD, max diameter of tumor.

superior vena cava replacement by artificial vessel is quite complicated. This article describes the technology of total superior vena cava reconstruction guided by preoperative 3D-CTBA.

\section{Methods}

\section{General data}

Five patients with superior vena cava system invade by the mediastinum tumor have been treated our medical centre. All the procedures were proved by the Ethics Committee of Fujian Medical University Union Hospital (No. 2020KY009) and conducted in accordance with the Declaration of Helsinki (as revised in 2013). Written informed consent was obtained from all patients for publication of this manuscript and any accompanying images. All patients had evident symptoms of superior vena cava obstruction, such as facial edema, cervical edema, jugular vein engorgement, chest wall filled with venous collateral circulation. The preoperative central venous pressure (CVP) was 30 to $40 \mathrm{cmH}_{2} \mathrm{O}\left(1 \mathrm{cmH}_{2} \mathrm{O}=0.098 \mathrm{kpa}\right)$ when lying on the elbow. High-quality thoracic CT image was essential, while the thickness of each level was less than $1 \mathrm{~mm}$. Preoperative 3D-CTBA reconstruction of SVC, $\mathrm{LBCV}, \mathrm{RBCV}$ and tumor lesions on the basis of highquality thoracic CT indicated that SVC, LBCV and RBCV were partly or completely invaded and occluded. According to 3D-CTBA images, tumor size, local range of invasion, pathological type and vascular compression or obstructed points (SVC, LBCV and RBCV, beginning and ending position of each vessel) were accurately understood before the operation (Table 1).

\section{Methods of treatment}

\section{Preoperative planning}

With accurate knowledge of preoperative 3D-CTBA, namely tumor size, local invasion scope, the degree of vascular compression or blocked position (SVC, LBCV and $\mathrm{RBCV}$, beginning and ending site of each vessel invaded), the whole process of operation were planned. The "Stepby-Step" approach proposed by our medical center was applied for designing the whole operation (Figure 1). By the display of the 3D-CTBA image, the anesthetists, cardiac doctors, intensive medicine doctors and thoracic surgeons conducted a more efficient multidisciplinary discussion together (Figures 2,3). An accurate preoperative surgical diagnosis guided by $3 \mathrm{D}-\mathrm{CTBA}$ was made as follows: (I) the necessity of performing superior vena cava replacement; (II) the necessity of performing veno-venous bypass support from the left internal jugular vein to the left femoral vein; (III) the possibility of radical resection of invaded SVC, LBCV and RBCV and complete dissection of anterior mediastinal tumor in the light of the principle of "en-bloc"; (V) the choice of suitable materials, such as artificial blood vessels, bovine pericardium (5); (V) the length of the vessel which would be dissected; (VI) the method of vascular anastomosis.

\section{Operation}

The actual operations of "total superior vena cava artificial vascular replacement" were conducted according to the planning process shown in Figure 1: veno-venous bypass support from the left internal jugular vein to the left femoral vein was performed in advance. The CVP of the SVC and the systemic blood pressure were monitored. Considering 


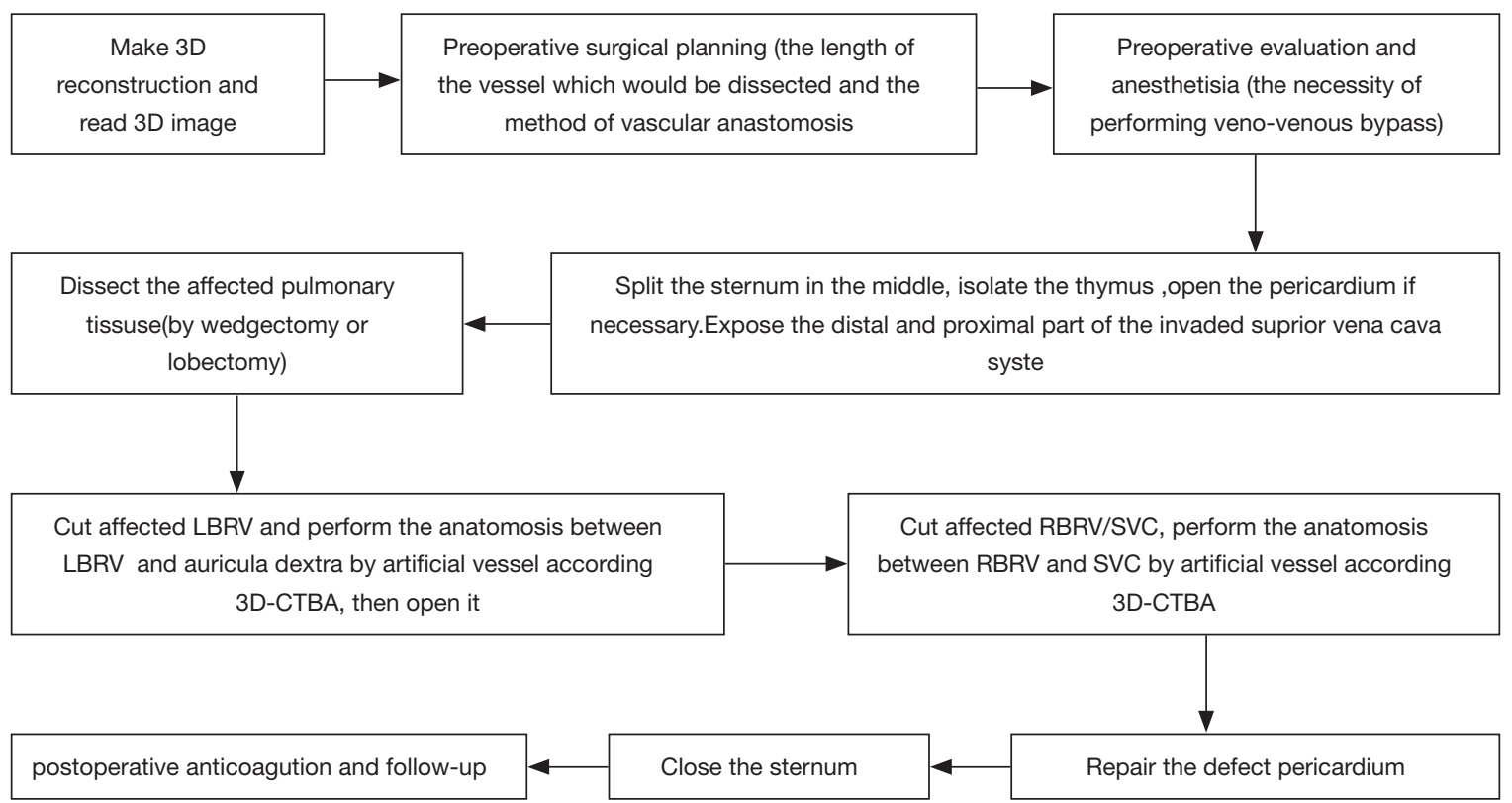

Figure 1 The "Step-by-Step" approach for SVC reconstruction. SVC, superior vena cava.

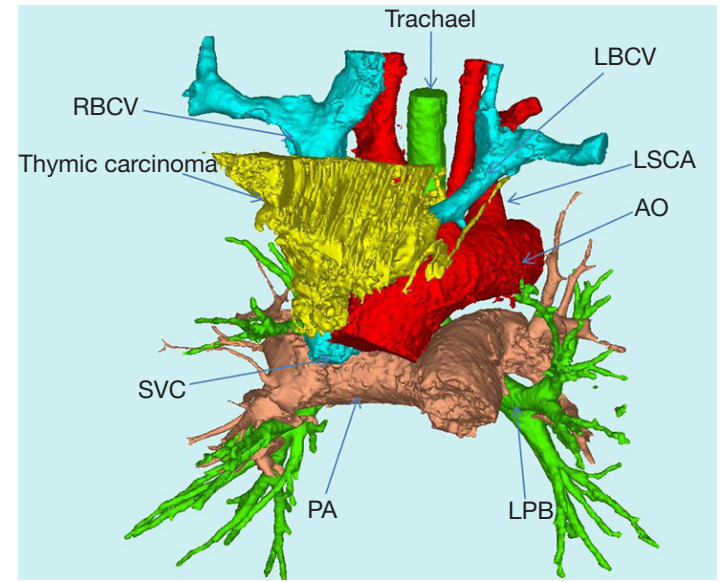

Figure 2 Anterior view of 3D-CTBA. SVC, superior vena cava; $\mathrm{LBCV}$, left brachiocephalic vein; RBCV, right brachiocephalic vein; AO, aorta; LSCA, left subclavian artery.

the level of CVP, two surgeries were performed with the veno-venous bypass opened. Once bypass was opened, CVP started to decrease, from a higher level of 30 to $40 \mathrm{cmH}_{2} \mathrm{O}$ to a lower level of 15 to $22 \mathrm{cmH}_{2} \mathrm{O}$.

The operation adopted a median incision in the chest. Since the tumor in the anterior mediastinum thymus was obviously invaded, the pericardium was often opened and dissected upward along the superior vena cava and ascending aorta. If the lung was invaded, a wedgectomy or lobectomy should be performed. The phrenic nerve (at least one side) was preserved during isolation. $\mathrm{LBCV}$ and $\mathrm{RBCV}$ were dissociated as long as possible to carry out the replacements by artificial vessel. The posterior separation of the tumor mainly focused on the three branches of the aortic arch (brachiocephalic artery, left common carotid artery and left subclavian artery) and trachea. During the operation, LBCV and RBCV were clamped in different stages (Figure 4). The duration of unilateral vascular occlusion should be less than 45 minutes while each end-to-end vascular anastomosis can be completed within 15 minutes (6).

All patients underwent total superior vena cava reconstruction with the assistance of preoperative 3D-CTBA. Among them, 4 underwent bilateral PTFE vascular replacement (Figure 4). One patient (case 3) had complete occlusion of LBCV with a distal vascular diameter of only $6.53 \mathrm{~mm}$. The distal diameter of RBCV was 16.99 $\mathrm{mm}$ (Figure 2) and there were only 10\# PTFE grafts. After preoperative $3 \mathrm{D}$ reconstruction and MDT discussion, it was decided to prepare bovine pericardial patch for reconstruction in advance. The CVP still fluctuated between 25 and $30 \mathrm{cmH}_{2} \mathrm{O}$ after the reconstruction with the $10^{\#}$ PTFE graft. When the bypass was closed, CVP increased to 35 to $40 \mathrm{cmH}_{2} \mathrm{O}$ immediately. The bypass had to be reopened. Two vascular end-to-end anastomoses (RBCV and artificial blood vessel, SVC and artificial blood 

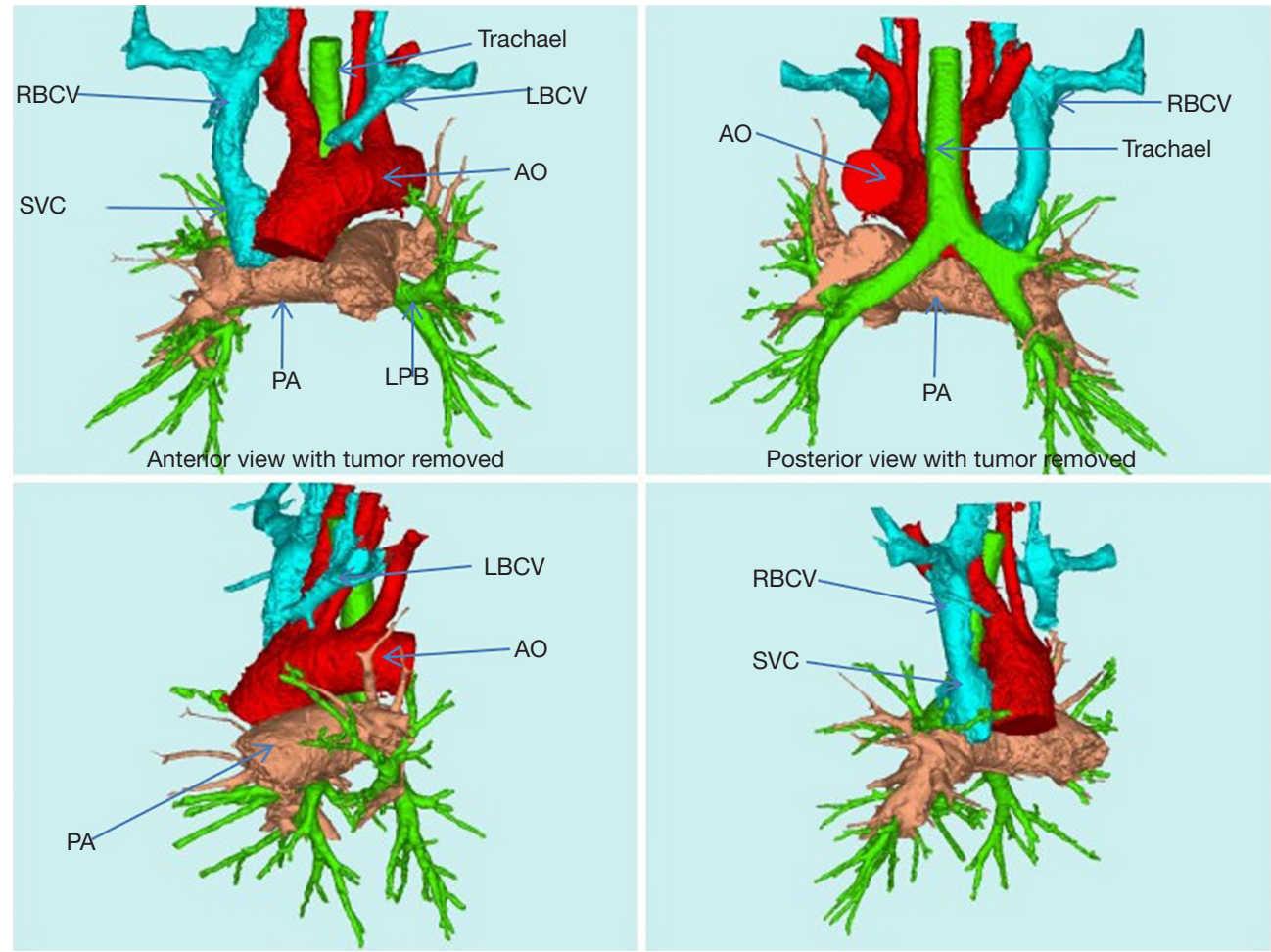

Figure 3 3D-CTBA images with tumor removed.

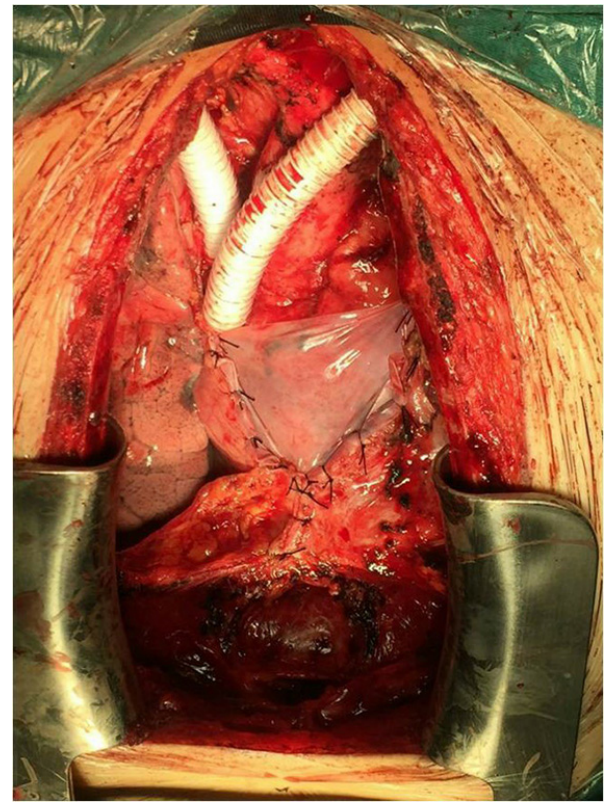

Figure 4 Bilateral PTFE vascular replacement for LBRV and RBRV. vessels) were demolished. The prepared bovine-pericardium patch was made into an artificial vessel (the diameter was about $2.0 \mathrm{~cm}$ ). Self-made artificial vessel connected RBCV and SVC (Figure 5). CVP decreased to 18 to $20 \mathrm{cmH}_{2} \mathrm{O}$ after re-reconstruction (5). After closing the bypass, CVP didn't rise obviously. All patients were transferred to the intensive care unit (ICU) and were discharged successfully.

\section{Follow-up}

Patents started to take warfarin for anticoagulant therapy immediately after surgery. APTT and INR were monitored through the dose of warfarin. INR was adjusted between 1.75 and 2.25. Chest CTA was conducted every three months within the first two years. 3D-CTBA was conducted to observe the recovery of four anastomotic stomas (Figure 6). The display of 3D-CTBA can clearly show whether there is stenosis or blockage.

\section{Statistical analysis}

All the data were analyzed by SPSS20.0 statistical software. The data was described by $\bar{x} \pm \mathrm{s}$ and the $t$-test were used for statistical analysis. The difference was statistically 


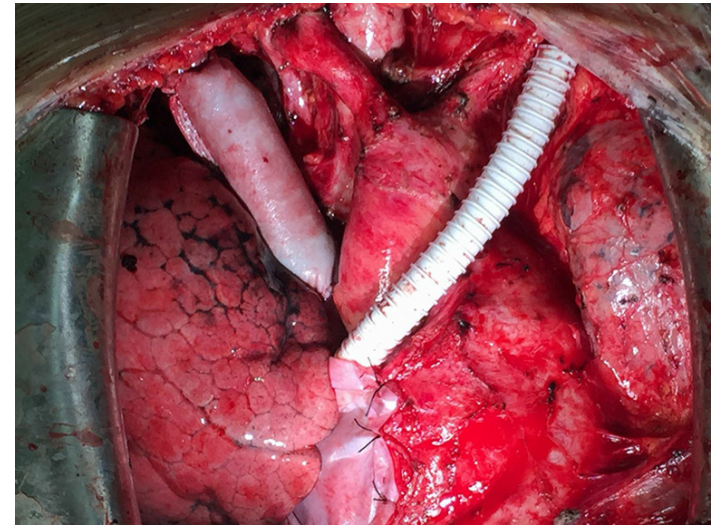

Figure 5 LBRV was replaced by 10\# PTFE graft while RBRV and SVC were replaced by an artificial vessel made of bovinepericardium patch.

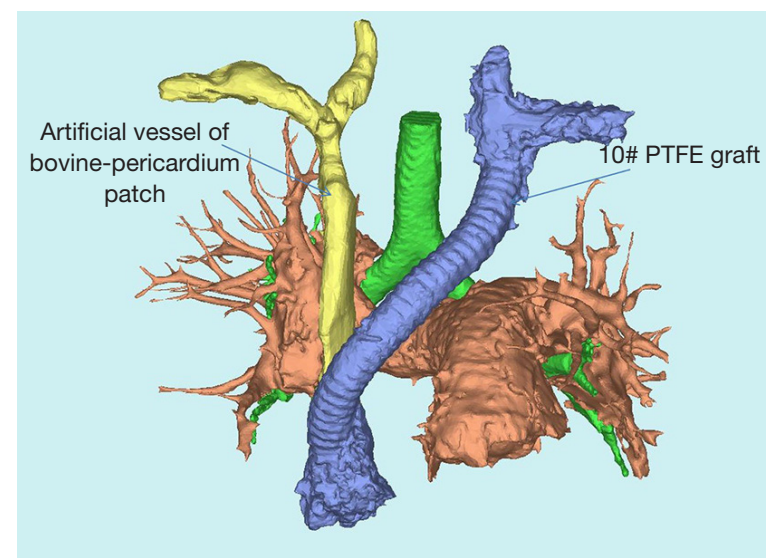

Figure 6 Postoperative 3D-CTBA showed the patency of two different artificial vessel (PEFE and bovine-pericardium graft) and the recovery of four anastomotic stomas.

significant at $\mathrm{P}<0.05$.

\section{Results}

There was no surgical mortality in this group. Symptoms of superior vena cava obstruction disappeared completely and the $\mathrm{CVP}$ was 12 to $20 \mathrm{~cm} \mathrm{H}_{2} \mathrm{O}$. Pulmonary infection occurred in two cases and radiotherapy or/and chemotherapy were selected for the 5 patients according to Masaoka stage after the surgery. The patients were followed up for 3 to 30 months and they were still alive now. The follow-up review showed that there was no recurrence of the tumor. They led a good quality of life (Table 2).

\section{Discussion}

The performance of radical resection of the operation for SVCS patients means satisfactory therapeutic effect (6). Although preoperative chest enhanced CT reveals adjacent relationship among mediastinal tumor, superior vena cava, aorta, and trachea, the "open and close" surgery or palliative resection may happen. Meanwhile, the necessity of superior vena cava replacement and the choice of artificial blood vessel depend on the probe result during the operation. Under such circumstances, we applied 3D-CTBA in the preoperative planning, the operation, postoperative followup of superior vena cava replacement. The key advantages of this technology could be summarized as follows.

\section{Accurate judgment assists radical dissection}

The assisting judgment of 3D-CTBA was achieved on the basis of clear images of CTA; Mask was precisely established in different positions or different organs. An accurate $3 \mathrm{D}$ image was reconstructed through the calculation of the precise mask. With the assistance of 3D-CTBA, the anatomic relationship between the tumor and the vessels could be observed by the simulated removal of tumor tissue (Figure 3). The proximal end of LBRC was completely invaded and blocked, while the RBCV and SVC were partly involved. By such assistance and judgment, the tightest point of tumor adhesion can be predicted before the operation and the dissociation during the operation would be dealt with more prudence. We would clamp the vessels in advance and open the bypass to reduce the amount of blood loss, improve the safety of the operation and dissect tumor radically.

3D-CTBA can identify the relationship among tumor, lung, trachea, aorta and its three branches. The relationship between mediastinal tumor and important organ can be shown more clearly in 3D-CTBA before surgery, comparing with conventional enhanced CT. To avoid unnecessary injury to vital organs, the dissociation during the operation would be dealt with more prudence under the guidance. At the same time, the radical resection of tumor tissue and other invaded organ could be predicted by $3 \mathrm{D}-\mathrm{CTBA}$. In this group, two patients underwent wedge resection in the 
Table 2 Intraoperative and postoperative data of this group

\begin{tabular}{lcccccc}
\hline Case & Time $(\mathrm{min})$ & Bleeding $(\mathrm{mL})$ & $\mathrm{ICU}$ stay $(\mathrm{d})$ & Total hospital stay $(\mathrm{d})$ & $\mathrm{CVP}^{*}\left(\mathrm{cmH}_{2} \mathrm{O}\right)$ & Follow-up time (month) \\
\hline 1 & 300 & 150 & 3 & 12 & 13 & 24 \\
2 & 270 & 150 & 3 & 11 & 12 & 13 \\
3 & 350 & 250 & 4 & 20 & 20 & 6 \\
4 & 400 & 200 & 5 & 18 & 11 & 30 \\
5 & 300 & 200 & 3 & 15 & 15 & 3 \\
$\mathrm{x}$ & 324 & 190 & 3.6 & 15.2 & 14.2 & 15.2 \\
$\mathrm{~s}$ & 45.86 & 37.41 & 0.8 & 3.43 & 3.19 & 10.34 \\
\hline
\end{tabular}

*, CVP when the vascular replacement was finished.

right upper lung.

\section{Accurate judgment assists vascular reconstruction}

The method of reconstruction is determined by accurate Judgment through preoperative 3D-CTBA. Before the surgery, the degree of vascular compression or the site of obstruction can be fully understood by 3D-CTBA reconstruction (Figure 3, the beginning and ending of superior vena cava, LBCV and RBCV obstructed). With the assistance of preoperative 3D-CTBA, an appropriate length of imported artificial vessels was selected to avoid the following two conditions: (I) large anastomotic tension caused by short artificial blood vessels and (II) thrombosis after anastomosis caused by the over length of artificial blood vessels. At the same time, external thread compression makes the artificial blood vessels less likely to be compressed by the lung and mediastinal tissue, preventing occlusion and deformation. However, mismatching of vascular caliber in reconstruction is prone to postoperative "blood steal", which leads to the decrease of blood flow in the vessels with fine caliber after the surgery. And further thrombosis and vascular occlusion may be induced. In the past, the heights and weights of the individual patients were the only criteria in choosing the size of artificial blood vessels. Generally, diameter of RBCV and SVC range from 1.4 to $1.0 \mathrm{~cm}$, diameter of LBCV 1.2 to $0.8 \mathrm{~cm}$. At present, when surgical planning is conducted through $3 \mathrm{D}-\mathrm{CTBA}$ reconstruction, the diameter, involved area and length of each blood vessel in the superior vena cava system can be fully measured, which promised the accurate selection of blood vessels. At the same time, excessive waste of medical resources will be avoided, reducing the financial burden of patients. Therefore, the application of 3D-CTBA makes the complex vascular reconstruction process more precise, flexible and controllable.

\section{Safety \& efficiency of the whole procedure}

Between June 2016 and December 2018, another 29 patients were diagnosed with the mediastinum tumor, which located close to superior vena cava system in the same medical group. 3D-CTBA was also conducted in these patients. Comparing with conventional CT images, $3 \mathrm{D}$ images promised accurate diagnosis that these twentynine patients did not need the replacement of superior vena cava before the operation. It was also proved that these mediastinal lesions could be isolated and dissected radically by VATS.

3D-CTBA also makes MDT and surgical cooperation more effective. With 3D-CTBA, all the doctors, including thoracic surgeon, anesthesiologist, ICU doctors, cardiac surgeon, and nurses would communicate effectively. In addition, patients were followed up by using 3D-CTBA so that the thoracic surgeon can follow-up the recovery of four anastomotic stomas (Figure 6). The display of 3D-CTBA can clearly show whether the anastomosis is too narrow or not. Thus, the surgeon would adjust the dosage of anticoagulant drugs according to coagulation function.

Although advantages of this technology were summarized as above, this favorable early clinical experience warrants further study.

\section{Acknowledgments}

Thanks Lin Gaojie for proofreading.

Funding: Supported by Program for Innovative Research Team in Science and Technology in Fujian Province 
University; Supported by Natural Science Foundation of Fujian Province (2016J01557) and Natural Science Foundation of Fujian Province (2018Y9034); Supported by 2019 Fujian Educational Research Projects (JAT190194).

\section{Footnote}

Conflicts of Interest: All authors have completed the ICMJE uniform disclosure form (available at http://dx.doi. org/10.21037/tcr-19-2249). The authors have no conflicts of interest to declare.

Ethics Statement: The authors are accountable for all aspects of the work in ensuring that questions related to the accuracy or integrity of any part of the work are appropriately investigated and resolved. The study was approved by Institutional Ethics Committee of Fujian Medical University Union Hospital (No.2020KY009) and conducted in accordance with the Declaration of Helsinki (as revised in 2013). Written informed consent was obtained from all patients for publication of this manuscript and any accompanying images.

Open Access Statement: This is an Open Access article distributed in accordance with the Creative Commons Attribution-NonCommercial-NoDerivs 4.0 International License (CC BY-NC-ND 4.0), which permits the noncommercial replication and distribution of the article with the strict proviso that no changes or edits are made and the original work is properly cited (including links to both the formal publication through the relevant DOI and the license). See: https://creativecommons.org/licenses/by-nc-nd/4.0/.

\section{References}

1. Sun Y, Gu C, Shi J, et al. Reconstruction of mediastinal vessels for invasive thymoma: a retrospective analysis of 25 cases. J Thorac Dis 2017;9:725-33.

2. Nakano T, Endo S, Kanai Y, et al. Surgical outcomes after superior vena cava reconstruction with expanded polytetrafluoroethylene grafts. Ann Thorac Cardiovasc Surg 2014;20:310-5.

3. Eguchi T, Takasuna K, Kitazawa A, et al. Threedimensional imaging navigation during a lung segmentectomy using an iPad. Eur J Cardiothorac Surg 2012;41:893-7.

4. Oizumi H, Kanauchi N, Kato H, et al. Anatomic thoracoscopic pulmonary segmentectomy under 3-dimensional multidetector computed tomography simulation: a report of 52 consecutive cases. J Thorac Cardiovasc Surg 2011;141:678-82.

5. D'Andrilli A, De Cecco CN, Maurizi G, et al. Reconstruction of the superior vena cava by biologic conduit: assessment of long-term patency by magnetic resonance imaging. Ann Thorac Surg 2013;96:1039-45.

6. Maurizi G, Poggi C, D'Andrilli A, et al. Superior Vena Cava Replacement for Thymic Malignancies. Ann Thorac Surg 2019;107:386-92.
Cite this article as: Zhang Z, Lin J, Chai T, Kang M, Chen W, Qiu H, Zou Z, Gao L, Yang C, Zhu J. Total superior vena cava reconstruction guided by preoperative three-dimensional (3D)computed tomography bronchography and angiography. Transl Cancer Res 2020;9(9):5411-5417. doi: 10.21037/tcr-19-2249 